\title{
Relationship between CD177 and the vasculogenic mimicry, clinicopathological parameters, and prognosis of epithelial ovarian cancer
}

\author{
Jing Jiang", Yan Chen", Miao Zhang, Honggui Zhou, Hong Wu \\ Department of Gynecology and Obstetrics, The Affiliated Hospital of North Sichuan Medical College, Nanchong, China \\ Contributions: (I) Conception and design: J Jiang, Y Chen; (II) Administrative support: M Zhang; (III) Provision of study materials or patients: J Jiang, \\ Y Chen, H Zhou, H Wu; (IV) Collection and assembly of data: All authors; (V) Data analysis and interpretation: J Jiang, M Zhang, H Zhou, H Wu; (VI) \\ Manuscript writing: All authors; (VII) Final approval of manuscript: All authors. \\ \#These authors contributed equally to the work and share first authorship. \\ Correspondence to: Jing Jiang. Department of Gynecology and Obstetrics, The Affiliated Hospital of North Sichuan Medical College, 63 Wenhua \\ Road, Shunqing District, Nanchong, China. Email: JingJiangSC@126.com.
}

\begin{abstract}
Background: To explore the relationship between CD177 and the vasculogenic mimicry (VM), clinicopathological parameters, and prognosis of epithelial ovarian cancer.

Methods: Tumor tissue samples and clinicopathological data were collected from 98 patients with epithelial ovarian cancer. The expression of CD177 in tumor tissues was detected by immunohistochemical streptavidin-peroxidase conjugate (SP) method, while the VM structure in tumor tissues was identified by CD31/periodic acid-Schiff (PAS) double staining in order to analyze the relationship between CD177, VM, clinicopathological parameters, and the prognosis of epithelial ovarian cancer.

Results: The proportion of the positive expression of CD177 $\left(\mathrm{CD} 177^{+}\right)$in 98 ovarian cancer tissues was higher than that of the negative expression of $\mathrm{CD} 177\left(\mathrm{CD} 177^{-}\right)(65.31 \%$ vs. $34.69 \%, \mathrm{P}<0.05)$. Univariate analysis showed that $\mathrm{CD} 177^{+}$was associated with VM formation, tumor differentiation degree, tumor diameter, tumor stages, and platinum sensitivity $(\mathrm{P}<0.05)$, and was not associated with age, tumor types, or lymph node metastasis $(\mathrm{P}>0.05)$. Correlation analysis showed that $\mathrm{CD} 177^{+}$was positively correlated with VM formation, tumor differentiation degree, tumor diameter, and tumor stages $(\mathrm{P}<0.05)$, and was negatively correlated with platinum sensitivity $(\mathrm{P}<0.05)$. Kaplan-Meier survival analysis showed that the survival time of $\mathrm{CD} 177^{+}$patients was significantly shorter than that of $\mathrm{CD} 177^{-}$patients $(\mathrm{P}<0.05)$.

Conclusions: $\mathrm{CD} 177^{+}$is associated with the tumor malignancy of patients with epithelial ovarian cancer, and may participate in the formation of VM structure in epithelial ovarian cancer tissues. It can thus serve as important indicator for the prognosis of patients.
\end{abstract}

Keywords! CD177; epithelial ovarian cancer; vasculogenic mimicry (VM); pathological parameters; prognosis

Submitted Aug 28, 2020. Accepted for publication Oct 28, 2020.

doi: 10.21037/apm-20-1825

View this article at: http://dx.doi.org/10.21037/apm-20-1825

\section{Introduction}

Ovarian cancer is one of the three major malignant tumors that occurs in female reproductive organs. The pathogenesis of ovarian cancer is still inconclusive, mainly with three theories. The incidence of ovarian cancer has been increasing each year, and its mortality has always ranked first in gynecological malignancies. Among all the ovarian cancers, epithelial ovarian cancer is the most common disease, and can seriously threaten the life of female patients (1). Epithelial ovarian cancer has an insidious onset, rapid progress. Pathological diagnosis is a common clinical method, but it is invasive operation, so it is 
lack of effective early diagnosis methods in clinical practice, Nearly $70 \%$ of patients are already in the advanced stage when the carcinomas are initially discovered. Furthermore, the treatment effect and prognosis of epithelial ovarian cancer are quite poor (2). Therefore, progress into understanding the pathogenesis and disease progression of epithelial ovarian cancer has been both critical and challenging in gynecological malignancies. The growth of tumor cells depends on blood supply. In addition to blood vessels, vasculogenic mimicry (VM) is an integral blood supply structure that maintains tumor growth (3). Previous studies have shown that cancer stem cells (CSCs) are closely related to the formation of VM (4). CD177 is an important neutrophil gene that encodes the neutrophil, NB1 glycoprotein, and acts as a molecular marker for CSCs (5). A large number of studies have found that CD177 is closely related to the neovascularization of epithelial ovarian cancer tumor tissues, suggesting that CD177 may be associated with the formation of VM in epithelial ovarian cancer tissue and may participate in the occurrence and development of epithelial ovarian cancer. In this study, CD177-positive expression $\left(\mathrm{CD} 177^{+}\right)$was detected in epithelial ovarian cancer tissue samples with complete clinical data, and the relationship between CD177 and the vascular VM, clinicopathological parameters, and prognosis of epithelial ovarian cancer was explored. We present the following article in accordance with the REMARK reporting checklist (available at http://dx.doi.org/10.21037/apm-20-1825).

\section{Methods}

\section{Specimens information}

A total of 98 specimens of epithelial ovarian tumor tissues were collected by operation from June 2012 to June 2017. The inclusion criteria were as follows: (I) patients were diagnosed as epithelial ovarian cancer by pathological diagnosis; (II) the first operation mode was an ovarian cancer staging operation or a tumor reduction operation; (III) patients had not received radiotherapy, chemotherapy, or hormone drugs before the operation (6,7); (IV) patients were supplemented with platinum-based combined chemotherapy after operation. Meanwhile, the exclusion criteria were the following: (I) patients with other types of tumor; (II) patients with incomplete clinical data and follow-up data; (III) patients who received radiotherapy, chemotherapy, or hormone drugs before the operation. The ages of patients ranged from 19 to 74 years old, with an average age of $52.36 \pm 12.02$. Tumor types included 63 cases of serous adenocarcinoma, 8 cases of endometriallike carcinoma, and 27 cases of mucinous adenocarcinoma. According to the 2009 International Federation of Gynecology and Obstetrics (FIGO) staging systems (6), there were 20 cases in stage I, 8 cases in stage II, 48 cases in stage III, and 22 cases in stage IV. According to the grade of differentiation, there were 11 cases with high differentiation, 48 cases with medium differentiation, and 39 cases with low differentiation. There were 45 cases of lymph node metastasis and 43 cases without lymph node metastasis. Among the patients, 29 were platinum-resistant (i.e., relapsed within 6 months after the discontinuation of primary platinum-based chemotherapy) and 69 were platinum-sensitive (i.e., no relapse occurred within 6 months after the discontinuation of primary platinum-based chemotherapy). This study was approved by the Ethics Committee of our hospital. All procedures performed in this study involving human participants were in accordance with the Declaration of Helsinki (as revised in 2013). The informed consent was taken from all the patients.

\section{Immunobistochemical streptavidin-peroxidase conjugate (SP) method}

The epithelial ovarian tumor tissues were conventionally fixed in $4 \%$ paraformaldehyde and embedded in paraffin wax. Sections were made from a tissue-slicing machine to the thickness of $4 \mu \mathrm{m}$. After dewaxing and hydration, 3\% $\mathrm{H}_{2} \mathrm{O}_{2}$ was used to eliminate endogenous peroxidase activity. Then, the tissue antigen was repaired by microwave, and the blocking solution containing goat serum was flooded on sections at $37^{\circ} \mathrm{C}$ for 45 minutes. Subsequently, rabbit antihuman CD177 monoclonal antibody (Boster Biological Technology, Wuhan, China) was added to the sections and incubated overnight at $4{ }^{\circ} \mathrm{C}$. The next day, goat antirabbit immunoglobulin $\mathrm{G}$ (IgG) antibody (Boster Biological Technology, Wuhan, China) labeled with horseradish peroxidase was added to the sections and incubated at $37{ }^{\circ} \mathrm{C}$ for 30 minutes. After reacted with DAB solution, the stained tissue sections were observed under optical microscope (Olympus, Japan). The CD177 immunohistochemical staining of gastric stromal tumor was used as the positive control, and sections incubated with phosphate-buffered saline (PBS) instead of the primary antibody were used as the negative control.

The determination of $\mathrm{CD} 177^{+}$expression was proceeded as follows. The brownish yellow granules in the cytoplasm 
or the cell membrane were considered to indicate the positive expression of CD177. The results of the test were double blind-determined by two experienced pathologists. According to Formwitz semiquantitative integration method, five high magnification visual fields $(\times 400)$ were randomly selected. Then, the positive cells in each visual field were counted, and the score of positive expression rate was calculated. Scoring of positive cells was determined according to the proportion of positive cells to the total number of cells, and tabulated in the following manner: less than $10 \%=0$ point; $11-30 \%=1$ point; $31-70 \%=$ 2 points; over $70 \%=3$ points. Staining intensity of cells was determined as follow: $0=$ staining; $1=$ light yellow; $2=$ brownish yellow. The staining index of each tissue section was the product of $\mathrm{CD} 177^{+}$expression rate score and cell staining intensity. CD177 expression was positive when the staining index was higher than 2 points, and CD177 expression was negative $\left(\mathrm{CD} 177^{-}\right)$when the staining index was lower than or equal to 2 points

\section{CD31/periodic acid-Schiff (PAS) double staining}

Rabbit anti-human CD31 monoclonal antibody (Boster Biological Technology, Wuhan, China) was used for immunohistochemical staining (same as the detection of CD177). The sections were then oxidized in $0.5 \%$ periodic acid solution for 8-15 minutes, washed with distilled water for 2 minutes, and then mixed with periodic acid-Schiff (PAS) reagent (ZSGB-BIO, Beijing, China) for staining. After 15 minutes, the sections were washed with distilled water 3 times, for 1 minute each time. The sections were then stained with hematoxylin. Finally, following conventional dehydration with gradient alcohol and transparency with xylene, the sections were sealed with neutral gum. The sections were photographed by observation under an optical microscope.

The standards for VM structure judgment were as follows. In a VM-positive (CD31-negative, PAS-positive) structure, the tumor cells with negative CD31 staining constitute the lumen-like structure. PAS-positive substances are attached to the surrounding, while no CD31-positive endothelial cells are found, and some lumens can be seen with red blood cells, platelets, etc. Meanwhile, in VMnegative (CD31-positive, PAS-positive) structure, tumor central necrosis is rarely seen around the VM. The inner wall of the blood vessel is lined by CD31-positive endothelial cells, which are brownish yellow, and PAS staining is positive. The results of the test were double- blind-determined by two experienced pathologists using the above-described standards.

\section{Follow-up}

All patients were followed up by outpatient service, telephone, or e-mail. The follow-up period was from June 2012, to December 2018. The clinicopathological data of all patients were recorded, including age, pathological tissue type, tumor tissue staging, tumor tissue differentiation, lymph node metastasis, and chemosensitivity. For patients who died during the follow-up, the time of death was recorded; for those who were lost to follow-up, the last follow-up date was recorded.

\section{Statistical analysis}

SPSS 20.0 software was performed for the statistical analysis. Frequency was used to express the counting data, and $\chi^{2}$ test was used to evaluate the differences. The correlation between CD177 and pathological data of patients was examined by Spearman's rank correlation. The Kaplan-Meier method was used for survival analysis while log-rank test was used to compare the survival curves. The difference was considered statistically significant with a $\mathrm{P}$ value $<0.05$.

\section{Results}

\section{Positive expression of CD177 in epithelial ovarian cancer}

The positive expression of $\mathrm{CD} 177\left(\mathrm{CD} 177^{+}\right)$was shown as brown-yellow particles in the nucleus, as shown in Figure 1. From a total of 98 cases of ovarian cancer, there were 64 $\mathrm{CD} 177^{+}$cases $(65.31 \%)$, and $34 \mathrm{CD} 177^{-}$cases $(34.69 \%)$. $\mathrm{CD} 177^{+}$patients were significantly more common than $\mathrm{CD} 177^{-}$patients $(\mathrm{P}<0.05)$.

\section{The relationship between $C D 177^{+}$expression and the $V M$ and clinicopathological parameters in epithelial ovarian cancer}

In ovarian cancer tissues, ovarian cancer cell focally expresses PAS positive substance, forms a lumen sample structure. The PAS positive substance isolated tumor cells from the lumen, and no CD31 positive was lined by endothelial cells. There were red blood cells in the part of the lumen, with no hemorrhage around lumen, no necrosis 
and inflammatory cells infiltration, indicating to be the VM, as shown in Figure 2. The positive expression of CD177 in epithelial ovarian cancer was correlated with VM formation, tumor differentiation, tumor diameter, tumor stage, and platinum sensitivity $(\mathrm{P}<0.05)$, but not with age, tumor type, or lymph node metastasis $(\mathrm{P}>0.05)$, as shown in Table 1 .

\section{Correlation analysis of CD177 expression with the VM and clinicopathological parameters in epithelial ovarian cancer}

As shown in Table 2, the positive expression of CD177 in epithelial ovarian cancer was positively correlated with VM formation, tumor differentiation, tumor diameter, and tumor stages $(\mathrm{P}<0.05)$, and negatively correlated with

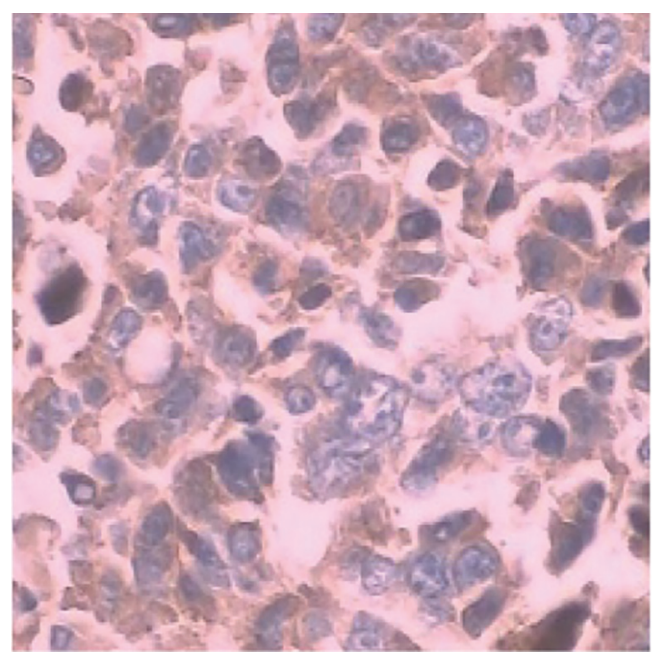

Figure 1 Expression of CD177 in ovarian cancer tissues detected by immunohistochemistry $(\times 400)$. platinum sensitivity $(\mathrm{P}<0.05)$.

\section{Relationship between survival rate and $C D 177^{+}$expression in epithelial ovarian cancer}

In all, 98 patients with epithelial ovarian cancer were followed up for 8 to 72 months. During the follow-up, 24 cases were lost, 43 cases died, and the mortality rate was $43.88 \%$. The survival curve of patients was drawn according to the expression of CD177. The median survival time of the $\mathrm{CD} 177^{-}$group was 71 months, and the median survival time of the $\mathrm{CD} 177^{+}$group was 32 months (Figure 3).

\section{Discussion}

Epithelial ovarian cancer is a common gynecological malignant tumor, accounting for $50-70 \%$ of ovarian cancer. Because it is difficult to detect in the early stage, the mortality rate ranks first among gynecological malignant tumors, and thus poses a considerable threat to the life of female patients (7). It is observed that during the rapid growth period of highly malignant epithelial ovarian cancer cells, the endothelium-dependent blood vessels formed by tumor angiogenesis cannot meet blood supply demand of tumor cells. Under these circumstances, the tumor cells will obtain the necessary nutrition through the construction of VM structures, and these structures will be connected with the endothelium-dependent blood vessels (8). The presence of VM will cause the epithelial ovarian cancer cells to come into direct contact with the blood circulation of the body. In turn, this causes the tumor cells to acquire higher invasion ability and increases the likelihood of drug resistance and recurrence, making

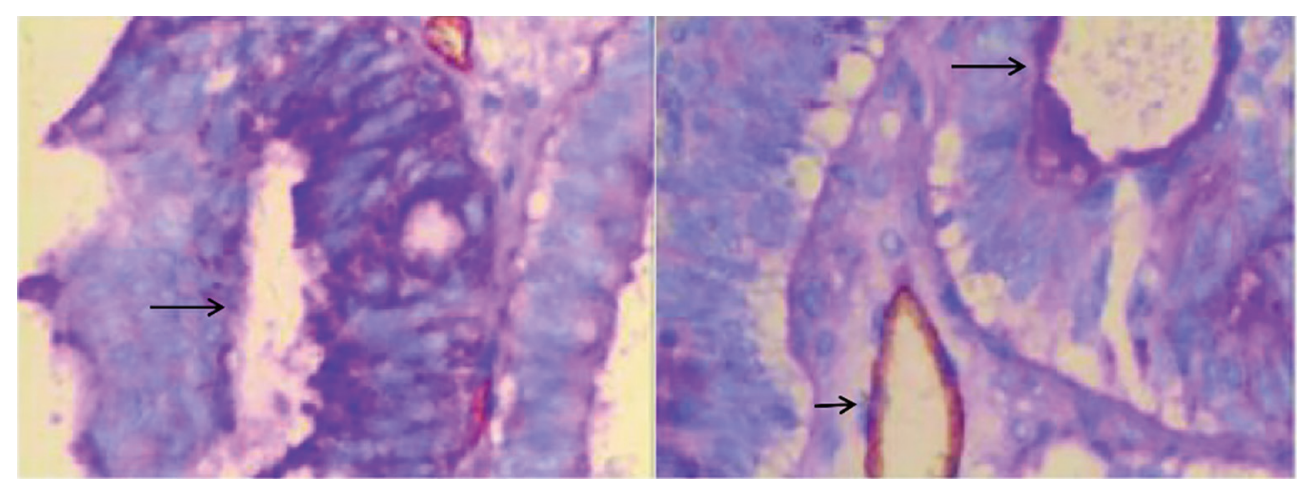

Figure 2 Double staining observation of VM CD31/PAS in ovarian cancer tissues $(\times 400)$. Long arrows indicated VM-positive CD31(-)/ PAS(+), and short arrows indicated VM-negative CD31(+)/PAS(+). 
Table 1 Relationship between $\mathrm{CD}_{177^{+}}$expression and the VM, and clinicopathological parameters in epithelial ovarian cancer

\begin{tabular}{|c|c|c|c|c|c|}
\hline Factors & Cases & $\mathrm{CD} 177^{-}$ & $\mathrm{CD} 177^{+}$ & $\chi^{2}$ & $\mathrm{P}$ \\
\hline $\mathrm{VM}^{+}$ & 43 & 11 & 32 & & \\
\hline $\mathrm{VM}^{-}$ & 55 & 28 & 27 & & \\
\hline Age (years) & & & & 0.615 & 0.433 \\
\hline$\geq 60$ & 30 & 9 & 21 & & \\
\hline Tumor types & & & & 0.096 & 0.956 \\
\hline Serous adenocarcinoma & 62 & 24 & 38 & & \\
\hline Endometrioid carcinoma & 16 & 6 & 10 & & \\
\hline High differentiation & 33 & 15 & 18 & & \\
\hline Medium differentiation & 27 & 8 & 19 & & \\
\hline Low differentiation & 38 & 4 & 34 & & \\
\hline Lymph node metastasis & & & & 1.654 & 0.198 \\
\hline Yes & 52 & 14 & 38 & & \\
\hline No & 46 & 18 & 28 & & \\
\hline Tumor diameter (cm) & & & & 5.280 & 0.022 \\
\hline$<5$ & 45 & 19 & 26 & & \\
\hline Stage IV & 22 & 2 & 20 & & \\
\hline Platinum sensitivity & & & & 6.907 & 0.009 \\
\hline Sensitive & 69 & 34 & 35 & & \\
\hline Resistant & 29 & 6 & 23 & & \\
\hline
\end{tabular}

VM, vasculogenic mimicry.

it difficult for antiangiogenesis treatment to completely block tumor blood vessels, which significantly worsens the prognosis and survival of patients (9).

CSCs are types of tumor cells that can be reproduced or regenerated. They are characterized by their small number of cells and their abilities of infinite proliferation, differentiation, and high tumorigenicity. They can be successfully transplanted into different species, presenting the same cell phenotype as the original tumor cells (10). Wang and his colleagues (11) found that CSCs were significantly related to the occurrence, invasion, metastasis, recurrence, and drug resistance of epithelial ovarian cancer. CSCs not only participate in the formation of tumor blood vessels, but also the formation of tumor 
Table 2 Correlation analysis of CD177 expression with VM and clinicopathological parameters in epithelial ovarian cancer

\begin{tabular}{lcc}
\hline Factors & $\mathrm{r}$ & $\mathrm{P}$ \\
\hline VM formation & 0.750 & $<0.05$ \\
Tumor differentiation & 0.653 & $<0.05$ \\
Tumor diameter & 0.574 & $<0.05$ \\
Tumor stages & 0.521 & $<0.05$ \\
Platinum sensitivity & -0.623 & $<0.05$ \\
\hline
\end{tabular}

VM, vasculogenic mimicry.

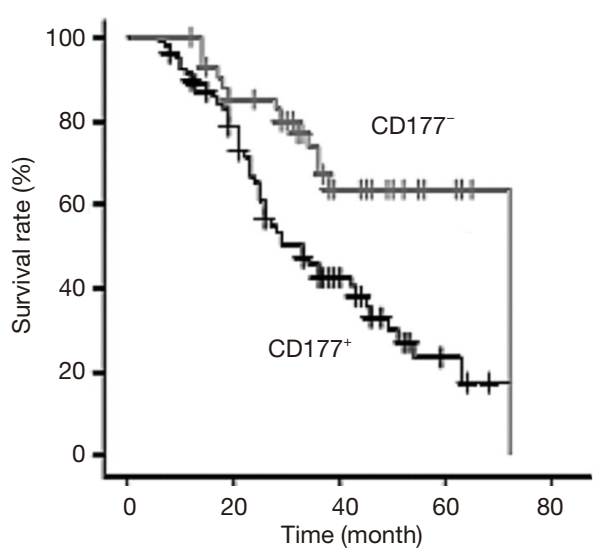

Figure 3 Survival curves of $\mathrm{CD} 177^{+}$and $\mathrm{CD} 177^{-}$patients.

cell VM structure through signal transduction pathway, cell shaping, and horizontal differentiation. CD177 is a widely studied CSC marker, which can participate in signal transduction, gene expression, cell proliferation, differentiation, and neovascularization, suggesting that the expression of CD177 in tumor tissue is related to the formation of $\mathrm{VM}$ and the occurrence and development of tumor $(12,13)$.

In this study, 98 epithelial ovarian cancer tissues were tested. CD $177^{+}$expression (64 cases) was significantly more abundant than $\mathrm{CD} 177^{-}$expression (34 cases). Furthermore, 64 epithelial ovarian cancer tissues were found, suggesting that CD177 was related to the occurrence and development of epithelial ovarian cancer, which is consistent with the published literature. In 43 cases of epithelial ovarian cancer with VM structure, 32 were CD $177^{+}$, while only 11 of these cases were $\mathrm{CD} 177^{-}$, indicating that CD177-mediated occurrence and development of ovarian cancer may be related to its involvement in VM formation. Yang et al. (14) also confirmed that $\mathrm{CD} 177^{+}$expression in ovarian cancer cells is closely related to the formation of tumor cell VM, invasion, and metastasis, which is consistent with the results of this study.

In order to further explore the relationship between CD177 in epithelial ovarian cancer and clinical pathological data, this study retrospectively collected various clinical pathological data of patients from each specimen source. Studies have shown that the positive expression of CD177 in epithelial ovarian cancer tissues is positively correlated with tumor differentiation grades, tumor diameter, and tumor stage. The proportion of $\mathrm{CD} 177^{+}$cells will be higher when the differentiation degree, tumor stage, and tumor diameter are greater, suggesting that the positive expression of CD177 in epithelial ovarian cancer tissues is related to the degree of tumor malignancy. The more malignant the tumor is, more new blood vessels and nutrients are needed for the tumor tissue, and, as the corresponding VM structure is increased, so is the positive expression of CD177 (15).

Our study also found that the positive expression of CD177 in epithelial ovarian cancer tissue is negatively correlated with the patient's platinum sensitivity, suggesting that positive expression of CD177 will reduce the patient's sensitivity to platinum drugs and induce the development of drug resistance. Moreover, the drug resistance will severely affect the treatment effect, leading to poor prognosis. It has been reported that CD177 can mediate various biological processes of tumor cells, and high expression of CD177 will induce tumor cells to proliferate, invade, and migrate through the activation of related signal pathways, thus affecting prognosis of patients (16). Subsequently, the Kaplan-Meier survival curve analysis in this study showed that the survival time and survival rate of $\mathrm{CD} 177^{+}$patients were significantly shorter and lower than those of CD177patients, suggesting that positive expression of CD177 would significantly reduce the survival rate of patients with epithelial ovarian cancer, leading to poor prognosis, which is consistent with the above studies.

In conclusion, positive CD177 expression may be related to the malignant degree of epithelial ovarian cancer, and may participate in the formation of VM structure in epithelial ovarian cancer, which is also is an important indicator of prognosis. The results thus suggest that CD177 is a potential clinical target for ovarian cancer. However, due to the limited sample size and short study time in this study, whether CD177 is related to the long-term prognosis of epithelial ovarian cancer patients and the relevant mechanism of its role in epithelial ovarian cancer is still 
unknown, and further study should be carried out in the further multi-center large sample trials.

\section{Acknowledgments}

Funding: None.

\section{Footnote}

Reporting Checklist: The authors have completed the REMARK reporting checklist. Available at http://dx.doi. org/10.21037/apm-20-1825

Data Sharing Statement: Available at http://dx.doi. org/10.21037/apm-20-1825

Conflicts of Interest: All authors have completed the ICMJE uniform disclosure form (available at http://dx.doi. org/10.21037/apm-20-1825). The authors have no conflicts of interest to declare.

Ethical Statement: The authors are accountable for all aspects of the work in ensuring that questions related to the accuracy or integrity of any part of the work are appropriately investigated and resolved. This study was approved by the Ethics Committee of our hospital. All procedures performed in this study involving human participants were in accordance with the Declaration of Helsinki (as revised in 2013). The informed consent was taken from all the patients.

Open Access Statement: This is an Open Access article distributed in accordance with the Creative Commons Attribution-NonCommercial-NoDerivs 4.0 International License (CC BY-NC-ND 4.0), which permits the noncommercial replication and distribution of the article with the strict proviso that no changes or edits are made and the original work is properly cited (including links to both the formal publication through the relevant DOI and the license). See: https://creativecommons.org/licenses/by-nc-nd/4.0/.

\section{References}

1. Reid BM, Permuth JB, Sellers TA. Epidemiology of ovarian cancer: a review. Cancer Biol Med 2017;14:9-32.

2. Peng $\mathrm{P}$, Zhang $\mathrm{W}$, Cao D, et al. The proteomic comparison of peripheral circulation-derived exosomes from the epithelial ovarian carcinoma (EOC) patients and
non-EOC subjects. Transl Cancer Res 2019;8:452-65.

3. Yao L, Zhang D, Zhao X, et al. Dickkopf-1-promoted vasculogenic mimicry in non-small cell lung cancer is associated with EMT and development of a cancer stemlike cell phenotype. J Cell Mol Med 2016;20:1673-85.

4. Liu TJ, Sun BC, Zhao XL, et al. CD133+ cells with cancer stem cell characteristics associates with vasculogenic mimicry in triple-negative breast cancer. Oncogene 2013;32:544-53.

5. Eulenberg-Gustavus C, Bähring S, Maass PG, et al. Gene silencing and a novel monoallelic expression pattern in distinct CD177 neutrophil subsets. J Exp Med 2017;214:2089-101.

6. Fan D, Lu YM. Expression of TRPS1 in epithelial ovarian cancer and the relationship with clinicopathological parameters. Maternal \& Child Health Care of China 2018;33:5229-32.

7. Huang JD. Expression of CD133 and Mir-145 in ovarian epithelial carcinoma, their clinicopathological characteristics and prognosis. Chinese Remedies \& Clinics 2019;19:1972-5.

8. Zhao P, Hong JJ, Chen X, et al. Clinical significance of circulating tumor cells detection from peripheral blood of patients with epithelial ovarian cancer. Journal of Modern Laboratory Medicine 2017;32:50-1.

9. Zou J, Liu L, Wang Q, et al. Downregulation of miR429 contributes to the development of drug resistance in epithelial ovarian cancer by targeting ZEB1. Am J Transl Res 2017;9:1357-68.

10. Gao S, Fan C, Huang H, et al. Effects of HCG on human epithelial ovarian cancer vasculogenic mimicry formation in vivo. Oncol Lett 2016;12:459-66.

11. Wang $Y$, Liu $P$, Wang $X$, et al. Role of X-linked inhibitor of apoptosis-associated factor-1 in vasculogenic mimicry in ovarian cancer. Mol Med Rep 2017;16:325-30.

12. Raggi C, Mousa HS, Correnti M, et al. Cancer stem cells and tumor-associated macrophages: a roadmap for multitargeting strategies. Oncogene 2016;35:671-82.

13. Sun B, Zhang D, Zhao N, et al. Epithelial-to-endothelial transition and cancer stem cells: two cornerstones of vasculogenic mimicry in malignant tumors. Oncotarget 2017;8:30502-10.

14. Yang B, Yan X, Liu L, et al. Overexpression of the cancer stem cell marker CD177 predicts poor prognosis in epithelial ovarian cancer patients: evidence from metaanalysis. Oncotargets \& Therapy 2017;10:2951-61.

15. Stemberger-Papić S, Vrdoljak-Mozetic D, Ostojić DV, et al. Expression of CD133 and CD117 in 64 Serous Ovarian 
Cancer Cases. Coll Antropol 2015;39:745-53.

16. Han F, Liu HQ, Wang SY. Expression of CD117, MITF and NAT10 and their prognostic values in sinonasal mucosal melanoma. Zhonghua Bing Li Xue Za Zhi.
2018;47:931-5.

(English Language Editor: J. Gray)

Cite this article as: Jiang J, Chen Y, Zhang M, Zhou H, Wu H. Relationship between CD177 and the vasculogenic mimicry, clinicopathological parameters, and prognosis of epithelial ovarian cancer. Ann Palliat Med 2020;9(6):3985-3992. doi: 10.21037/ apm-20-1825 